\title{
Self-Healing Material Design and Optimization for Soft Robotic Applications ${ }^{\dagger}$
}

\author{
Joost Brancart $^{1,2^{*}}$, Seppe Terryn ${ }^{1,2}$, Ellen Roels ${ }^{2}$, Bram Vanderborght ${ }^{2}$, Guy Van Assche ${ }^{1}$ \\ 1 Physical Chemistry and Polymer Science, Department of Materials and Chemistry, Vrije Universiteit Brussel, Pleinlaan \\ 2, B-1050 Brussel, Belgium; guy.van.assche@ vub.be (G.V.); \\ 2 Robotics \& Multibody Mechanics, Department of Mechanical Engineering, Vrije Universiteit Brussel, Pleinlaan 2, B-1050 \\ Brussel, Belgium; bram.vanderborght@vub.be (B.V.); \\ * Correspondence: joost.brancart@ vub.be; \\ $\uparrow$ Presented at Materials Chemistry and Physics (Materials Chemistry 2020) - International e-Conference
}

Received: 16.09.2020; Revised: 20.09.2020; Accepted: 24.09.2020; Published: 27.09.2020

\begin{abstract}
The Diels-Alder reaction between furan and maleimide is the most studied example of reversible covalent chemistries for creating self-healing materials. While scientific articles reporting the synthesis of new reversible polymer networks are numerous, accurate knowledge of the reaction kinetics and thermodynamics of the dynamically reversible equilibrium reaction and the structure and property development of derived stimuli-responsive materials are less widespread. The requirements for the material properties and behavior become more stringent when designing materials for dedicated applications, such as soft robotic structures. Optima need to be sought between reasonably fast reaction kinetics for fast and efficient damage healing at moderate temperatures and mechanical strength and structural stability on the other hand. Stress relaxation is desired to make materials tougher, relieving stress before defects can grow into cracks and ultimately lead to failure, while creep can't be allowed. Recycling and reprocessing of materials are desirable from an ecological viewpoint, while the materials should also be able to withstand static and dynamic loading in a considerable range of environmental conditions. Accurate knowledge of the reaction kinetics and thermodynamics and an in-depth knowledge of structure-processing-property relations allow smart polymer network design with tailored stimuli-responsive behavior and use as self-healing materials for robotic applications.
\end{abstract}

Keywords: dynamic covalent networks; self-healing materials; soft robotic actuators.

(c) 2020 by the authors. This article is an open-access article distributed under the terms and conditions of the Creative Commons Attribution (CC BY) license (https://creativecommons.org/licenses/by/4.0/).

\section{Funding}

This research was funded by the Fonds Wetenschappelijk Onderzoek (FWO), postdoctoral fellowship 12W4719N, and predoctoral fellowship 1S84120N, and by the Horizon 2020 program of the European Union, FET Open RIA project SHERO with grant number 828818.

\section{Acknowledgments}

This research has no acknowledgment.

\section{Conflicts of Interest}

The authors declare no conflict of interest. 